\title{
Estudio retrospectivo de los principales sistemas orgánicos afectados en 435 caninos traumatizados (1996-2007)
}

\author{
GINO CATTANEO U., M. V. ${ }^{1}$, FRANCISCA BAHAMONDES G., M. V. ${ }^{1}$, ESTEFANÍA FLORES P., M. V. ${ }^{1}$, y \\ ANDRÉS BASTÍAS P., M. V. ${ }^{1}$
}

1 Departamento Ciencias Clínicas, Facultad de Ciencias Veterinarias y Pecuarias.

\section{ABSTRACT \\ RETROSPECTIVE STUDY OF THE MAIN ORGANIC SYSTEM AFFECTED ON 435 INJURIED DOGS (1996-2007)}

In the present study analyzed the major organ systems affected in trauma patients. Resulting in the musculoskeletal system that corresponds to the main system affected a total of 435 canines.

Key words: Trauma, Organic System.

\section{RESUMEN}

Se realizo un estudio retrospectivo de 435 atenciones en caninos que sufrieron algún tipo de trauma, analizándose los principales sistemas orgánicos afectados. Se encontró que los principales daños afectaron al sistema músculo- esquelético y en segundo lugar al sistema tegumentario. Es considerablemente menor el porcentaje de presentación de otros sistemas afectados.

Palabras clave: Trauma, Sistema orgánico.

\section{INTRODUCCIÓN}

Trauma es una palabra griega cuyo significado es "herida". El término actual denota una injuria producida súbitamente por acción física o química. También esta palabra incluye el evento por el cual se produce la herida según Kolata (1981).

Las injurias traumáticas más comunes en veterinaria corresponden a los accidentes vehiculares, las quemaduras, las heridas provocadas por: las armas, otros animales y por los humanos. El trauma es la principal causa de muerte en los animales pequeños, y puede disminuir con una metodología sistemática y organizada que incluye una evaluación cuidadosa del paciente, una resucitación agresiva, un tratamiento definitivo y un buen equipo médico entrenado en medicina 
de emergencia de acuerdo a Wingfield (2002).

Kolata y Dudley (1975) en 600 caninos atropellados, obtuvo como primera región injuriada la zona de las extremidades, en segundo lugar la cabeza y luego la pelvis. A su vez, en el estudio que realizó al año siguiente con 871 caninos traumatizados en que asoció motivo de trauma con localización de la injuria, arrojó resultados similares a este estudio, ya que estableció daño principalmente en las extremidades en los atropellos, al igual que en los traumas de causa desconocida. En las mordeduras se observó que la cabeza es la que se ve principalmente dañada, probablemente debido a ser la zona de cercanía inmediata en las peleas.

Esta investigación tuvo por objetivo definir cuales son los sistemas orgánicos más afectado en pacientes que han sido sometidos a un trauma y de esta forma los resultados sean utilizado como antecedente al momento de realizar un examen clínico a un paciente traumatizado.

\section{MATERIAL Y MÉTODO}

El presente trabajo realiza una descripción epidemiológica de los sistemas orgánicos macroscópicamente involucrados en caninos traumatizados ingresados al Servicio de Cirugía Veterinaria de la Facultad de Cs. Veterinarias y Pecuarias de la Universidad de Chile (SCV), entre Enero del año 1996 hasta Diciembre del año 2007.

Se utilizó el registro de pacientes traumatizados ingresados al SCV desde el Enero del año 1996 hasta Diciembre del año 2007, desde el cuál se obtuvo 435 casos de caninos.

\section{RESULTADOS}

Al dividir el organismo en siete sistemas orgánicos se pudo detectar cuales de ellos eran los más afectados a partir del trauma. En el Gráfico 1 se observa el sistema orgánico que se vio mayormente afectado fue el músculoesquelético con un $54,7 \%$.

Un 21,9\% pertenece al sistema tegumentario. El sistema ocular fue afectado en un 7,5\% de los pacientes.

Los casos en que se involucró el sistema neurológico correspondieron al 4,9\%. El sistema respiratorio y digestivos obtuvieron un $4,1 \%$ cada
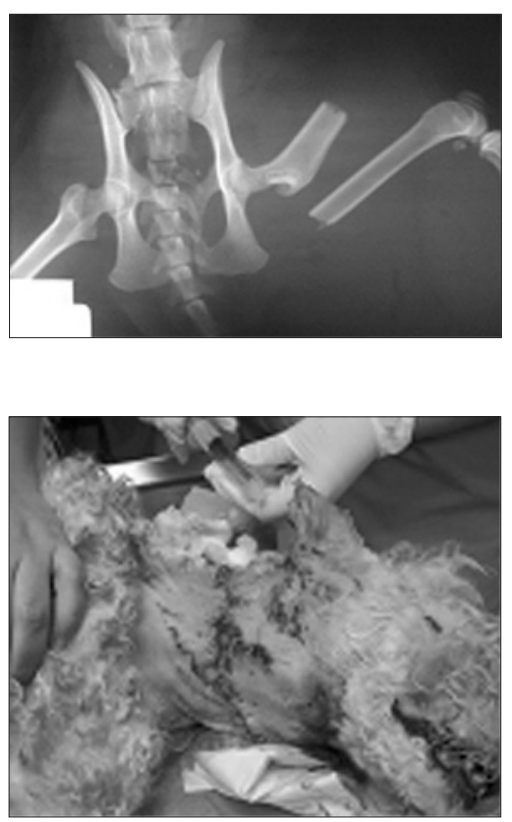

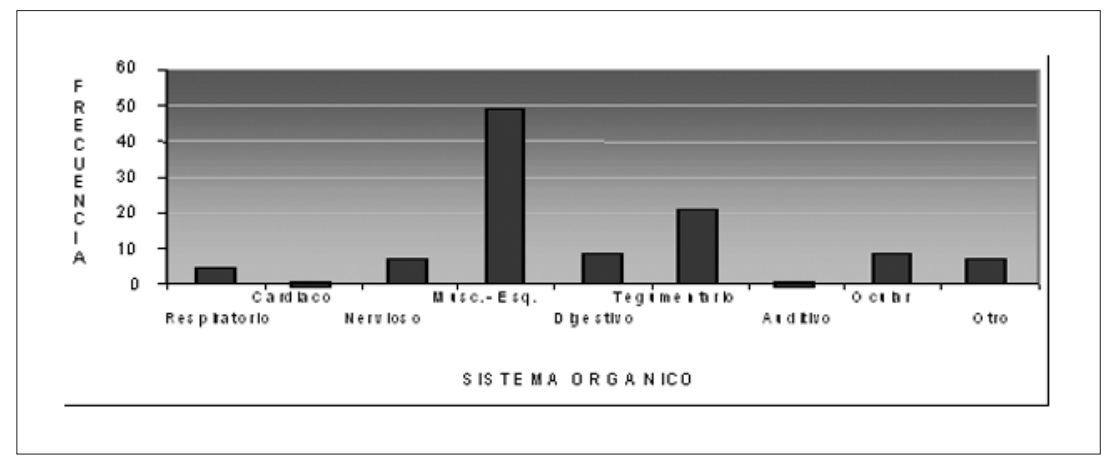

Gráfico 1. "Población canina según sistema involucrado". Servicio de Cirugía de Animales Pequeños. Universidad de Chile. Enero 1996 - Diciembre 2007. 

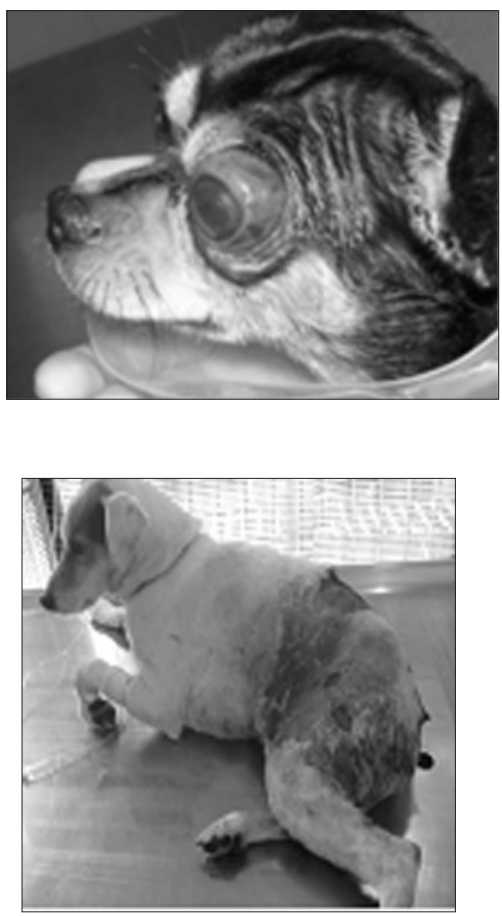

uno. Los sistemas orgánicos que se vieron menos afectados fueron el sistema cardiaco y sistema auditivo con $0,5 \%$ y $0,3 \%$ respectivamente. Un $1,9 \%$ correspondió a otros sistemas orgánicos no mencionados anteriormente.

\section{DISCUSIÓN}

El sistema músculo- esquelético fue el que se vio más afectado, junto con el tegumentario. Ambos sistemas en la mayoría de los casos no involucra un importante riesgo vital sin dejar de ser valiosa su evaluación. Es importante destacar que sólo se evaluó lesiones macroscópicas, no considerándose lesiones microscópicas asintomáticas. Estos resultados tienen como utilidad servir como antecedente para el médico veterinario al momento de realizar un examen clínico a un paciente traumatizado, teniendo en consideración cuales son los sistemas que en mayor porcentaje se ven afectados.

\section{REFERENCIAS}

1.- ARREDONDO R. 2003. Factores epidemiológicos en perros y gatos politraumatizados. Hospital Veterinario Universidad de Chile, Santiago. Memoria de título, Med. Vet. Universidad de Chile, Fac. de Cs. Vet. y Pecuarias, Santiago, Chile. 25-60.

2.- CRANE S. 1996 Epidemiología y mecanismos de los traumatismos en los animales de compañía. Cirugia de los Pequeños Animales. Tomo I. Editor Slatter, H.D. Editorial Masson. España.

3.- DROBATZ K. 2002. Global Approach to the Trauma Patient. In: The North American Veterinary Conference Small Animals and Exotics. Gainesville, U.S.A. 1216 Enero 2002. Eastern States veterinary Association. 130-132.

4.- GUERRERO R, GONZÁLEZ C, MEDINA E. 1981. Estudio de casos y controles retrospectivos. In: E. Wesley (Ed). Epidemiología. Fondo educativo Interamericano, Bogotá, Colombia. 101-111.

5.- KOLATA R, DUDLEY E. 1975. Motor vehicle accidents in urban dogs: A estudy of 600 cases. J Am Vet Med Assoc 167(8): 938-941.

6.- KOLATA R. 1981. Patterns and Incidence of Trauma. In: Archibald J.; Holt,J.Sokolovsky, V.Management of Trauma in Dogs and Cats. American Veterinary Publications, Inc. Santa Barbara, U.S.A. 11-20.

7.- WINGFIELD W. 2002. Equipping and Emergency/ Critical care veterinary Hospital In: Wingfield, W.; Raffe, M. (Eds) The Veterinary ICU Book, Teton NewMedia. Jackson Hole, U.S.A. 1174-1176. 